\title{
Addressing the dichotomy between individual and societal approaches to personalised medicine in oncology
}

Citation for published version (APA):

Salgado, R., Solit, D. B., Rimm, D. L., Bogaerts, J., Canetta, R., Lively, T., Lyerly, K., Span, P. N., Bateman-House, A., Makady, A., Bergmann, L., Nagai, S., Smith, C., Robson, M., Savage, M., Voest, E., Sweeney, C., Lambin, P., Thomas, M., ... IBCD-Faculty (2019). Addressing the dichotomy between individual and societal approaches to personalised medicine in oncology. European Journal of Cancer, 114, 128-136. https://doi.org/10.1016/j.ejca.2019.03.025

Document status and date:

Published: 01/06/2019

DOI:

10.1016/j.ejca.2019.03.025

Document Version:

Publisher's PDF, also known as Version of record

\section{Document license:}

Taverne

Please check the document version of this publication:

- A submitted manuscript is the version of the article upon submission and before peer-review. There can be important differences between the submitted version and the official published version of record.

People interested in the research are advised to contact the author for the final version of the publication, or visit the DOI to the publisher's website.

- The final author version and the galley proof are versions of the publication after peer review.

- The final published version features the final layout of the paper including the volume, issue and page numbers.

Link to publication

\footnotetext{
General rights rights.

- You may freely distribute the URL identifying the publication in the public portal. please follow below link for the End User Agreement:

www.umlib.nl/taverne-license

Take down policy

If you believe that this document breaches copyright please contact us at:

repository@maastrichtuniversity.nl

providing details and we will investigate your claim.
}

Copyright and moral rights for the publications made accessible in the public portal are retained by the authors and/or other copyright owners and it is a condition of accessing publications that users recognise and abide by the legal requirements associated with these

- Users may download and print one copy of any publication from the public portal for the purpose of private study or research.

- You may not further distribute the material or use it for any profit-making activity or commercial gain

If the publication is distributed under the terms of Article $25 \mathrm{fa}$ of the Dutch Copyright Act, indicated by the "Taverne" license above, 


\section{Current Perspective}

\section{Addressing the dichotomy between individual and societal approaches to personalised medicine in oncology}

Roberto Salgado ${ }^{\mathrm{a}, \mathrm{b}, *}$, David B. Solit ${ }^{\mathrm{c}}$, David L. Rimm ${ }^{\mathrm{d}, \mathrm{e}}$, Jan Bogaerts ${ }^{\mathrm{f}}$, Renzo Canetta ${ }^{g}$, Tracy Lively ${ }^{\text {h}}$, Kim Lyerly ${ }^{i}$, Paul N. Span ${ }^{j}$, Alison Bateman-House ${ }^{\mathrm{k}}$, Amr Makady ${ }^{1}$, L. Bergmann ${ }^{\mathrm{m}, \mathrm{n}}$, Sumimasa Nagai ${ }^{\circ}$, Chris Smith ${ }^{\mathrm{p}}$, Mark Robson ${ }^{\mathrm{c}}$, Mary Savage ${ }^{\mathrm{q}}$, Emile Voest ${ }^{\mathrm{r}}$, Christopher Sweeney ${ }^{\mathrm{s}}$, Philippe Lambin ${ }^{\mathrm{t}}$, Marlene Thomas ", Lyndsay Harris ', Denis Lacombe ${ }^{\mathrm{f}}$, Chistophe Massard ${ }^{v}$ on behalf of the IBCD-Faculty ${ }^{1}$

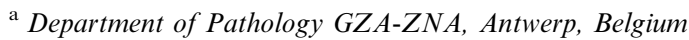

${ }^{\mathrm{b}}$ Division of Research, Peter Mac Callum Cancer Center, Melbourne, Australia

${ }^{c}$ Memorial Sloan Kettering Cancer Center, New York, NY, USA

${ }^{\mathrm{d}}$ Dept. of Pathology, Yale University School of Medicine, New Haven, CT, USA

${ }^{\mathrm{e}}$ Dept. of Medicine, Yale University School of Medicine, New Haven, CT, USA

${ }^{\mathrm{f}}$ EORTC Headquarters, Brussels, Belgium

${ }^{\mathrm{g}}$ Independent Consultant, Madison, CT, USA

${ }^{\mathrm{h}}$ National Cancer Institute, Bethesda, MD, USA

${ }^{\mathrm{i}}$ Duke University, Durham, NC, USA

${ }^{\mathrm{j}}$ Radiotherapy and OncoImmunology Laboratory, Department of Radiation Oncology, Radboud University Medical Center, Nijmegen, the Netherlands

${ }^{\mathrm{k}}$ Division of Medical Ethics, NYU Langone Health, New York, NY, USA

${ }^{1}$ The National Healthcare Institute (ZIN), Diemen, the Netherlands

${ }^{\mathrm{m}}$ Medical Clinic II, University Hospital Frankfurt, Germany

${ }^{\mathrm{n}}$ Ambulantes Krebszentrum Frankfurt, Germany

${ }^{\circ}$ Translational Research Center, The University of Tokyo Hospital and PMDA, Tokyo, Japan

${ }^{\mathrm{p}}$ CRUK Cambridge Institute, University of Cambridge, Cambridge, United Kingdom

${ }^{\mathrm{q}}$ Merck, Whitehouse Station, NJ, USA

${ }^{\mathrm{r}}$ The Netherlands Cancer Institute, Amsterdam, the Netherlands

${ }^{\mathrm{s}}$ Dana Farber Cancer Institute, Harvard Medical School, Boston, MA, USA

${ }^{\mathrm{t}}$ The D-Lab \& The M-Lab, Department of Precision Medicine, GROW Research Institute for Oncology, Maastricht, The Netherlands

${ }^{\mathrm{u}}$ Roche, Basel, Switzerland

${ }^{v}$ Institut Gustave Roussy, Paris, France

Received 20 March 2019; accepted 28 March 2019

Available online 3 May 2019

\footnotetext{
* Corresponding author: Department of Pathology GZA-ZNA, Antwerp, Belgium. E-mail address: roberto@salgado.be (R. Salgado).

${ }^{1}$ See Addendum.
} 


\section{KEYWORDS}

Biomarkers;

Evidence-driven

optimal health-care

delivery;

Molecular and

immunologic profiling;

Health technology

assessment;

Clinical trials

\begin{abstract}
Academic, industry, regulatory leaders and patient advocates in cancer clinical research met in November 2018 at the Innovation and Biomarkers in Cancer Drug Development meeting in Brussels to address the existing dichotomy between increasing calls for personalised oncology approaches based on individual molecular profiles and the need to make resource and regulatory decisions at the societal level in differing health-care delivery systems around the globe. Novel clinical trial designs, the utility and limitations of real-world evidence (RWE) and emerging technologies for profiling patient tumours and tumour-derived DNA in plasma were discussed. While randomised clinical trials remain the gold standard approach to defining clinical utility of local and systemic therapeutic interventions, the broader adoption of comprehensive tumour profiling and novel trial designs coupled with RWE may allow patient and physician autonomy to be appropriately balanced with broader assessments of safety and overall societal benefit.

(c) 2019 Published by Elsevier Ltd.
\end{abstract}

\section{Introduction}

A dichotomy exists between oncology approaches based on personalised molecular profiles and resource and regulatory decisions for societal needs. Broader adoption of tumour profiling, novel trial designs and realworld evidence (RWE) may facilitate balancing of patient/physician autonomy with safety concerns and broader societal benefit.

\section{Individual and societal approaches to personalised medicine in oncology}

Recent large-scale collaborative efforts to define the molecular changes that promote cancer formation and a deeper understanding of the host immune response have resulted in a rapidly expanding toolkit of targeted and immunological therapies. In many cases, these biologically guided therapies are significantly more effective and less toxic than traditional cytotoxic agents, often resulting in durable treatment responses in selected populations of advanced cancer patients as well as improved outcomes in resectable tumours (e.g. melanoma with immune-oncology and targeted agents).

Historically, the clinical evidence documenting the effectiveness of newer cancer therapies has been generated under the guidance of traditional drug approval pathways, most often through prospective randomised clinical trials. As many newer targeted therapies are likely to be effective in smaller subpopulations of patients, the necessity and relevance of this traditional drug development paradigm has recently been questioned. Anecdotal responses in the setting of off-label use, retrospective analyses of outlier responses or small cohort studies and even biologic proof-of-concept laboratory data have all been used as justification by oncologists and patients to request non-trial access to newer cancer drugs. These provider and patient demands for access to marketed or investigational drugs have led to increasing tension between point-of-care oncologists who may wish to pursue individualised treatment paradigms in the hope of providing benefit to individuals lacking effective standard treatment options and national regulatory authorities, such as the United States Food and Drug Administration (FDA), the European Medicines Agency (EMA) and the Japanese Pharmaceuticals and Medical Devices Agency (PMDA), charged with ensuring that unsafe or ineffective therapies are not approved. Finally, given the high cost of many novel cancer therapies, Health Technology Assessment (HTA) bodies advising government payers and/or private insurers often limit access to nonstandard treatment approaches because of uncertainties over clinical benefit or as a result of unfavourable cost-benefit analyses.

Despite scientific advances in our understanding of tumour biology, most regulatory approvals continue to apply to only a specific therapy, in a particular tumour type and clinical setting [1]. Although new regulatory approaches are emerging, their impact so far has been limited. For example, histology-agnostic clinical trials have emerged as a tool to assess the broader utility of a cancer therapeutic in populations defined by a biomarker rather than tumour type. These histologyagnostic trials or 'basket trials' are a recent innovation that allow for the testing of newer therapies in patients with a shared biologic process, for example, a specific mutation such as BRAF V600E or a mutational signature coupled with a targeted agent against that process. Recently, the FDA and PMDA used a tissue-agnostic clinical trial as the basis for the regulatory approval of pembrolizumab, a PD-1 targeted antibody, for solid tumour patients whose cancer are MSI-High or have DNA mismatch repair deficiency (MSI-H/-dMMR). EMA, however, has not approved pembrolizumab, and its perspective differs from that of the FDA. While pembrolizumab had previously been approved for other cancer indications, a single-arm tumour agnostic trial 
was useful for expanding the label to include MSI-H/dMMR patients of any cancer type. Similarly, a small basket trial led to accelerated FDA approval of larotrectinib (LOXO-101) for the treatment of patients with NTRK-fusion cancers (up to $1 \%$ of all solid tumours) of any primary site in the United States [2]. Larotrectinib thus represents the first oncology drug to receive a tumour agnostic indication at the time of initial regulatory approval. While these recent successes provide a roadmap for future histology agnostic drug development paradigms, at least in the United States, it remains unclear whether small basket trials will be sufficient to influence drug access policies dictated by regulatory bodies that require more rigorous evidence and HTA agencies which are charged with assessing the relative effectiveness and cost-effectiveness of tumour agnostic drugs as compared with current tumours-specific standards of care [3]. Notably, both of the aforementioned tumour agnostic indications were approved, absent a coapproval for a companion diagnostic test (CDx) in the United States, which has led to the emergence of numerous laboratory-derived tests for which the concordance between methods, e.g. next generation sequencing (NGS), fluorescence in situ hybridisation (FISH) and immunohistochemistry (IHC), and the optimal method for predicting drug response remains unclear. This has led to significant uncertainty among treating physicians as to the best methods by which to screen patients for MSI-H/-dMMR or NTRK fusions. Thus, in the absence of a single validated test, it is not clear whether some patients are being harmed by the use of inaccurate diagnostic tests for which analytical sensitivity and accuracy are unknown. This is not to infer that all laboratory developed tests (LDTs) are inaccurate, but rather that some labs benefit from the use of standardised closed-system kits. Given these concerns, the approval of drugs targeting MSI-H/dMMR and NTRK were coupled with postmarketing requirements for the development of companion diagnostic devices.

Given the increasingly complex nature of biomarkers and the need for assays that can rapidly and correctly identify the optimal patients for individual biologic therapies, there has been a recognised need for the development of analytically validated, robust, accurate and reproducible tests, a need which historically drove the regulatory recommendation for the development of drug-specific companion diagnostic tests. Some oncologists have, however, questioned the need for companies to develop companion diagnostics for all new cancer therapies, at least for those that target DNA-based alterations such as mutations, gene amplifications or fusions. As multigene NGS assays become increasingly accurate and cost effective, the necessity of single-gene companion diagnostics that may increase cost, delay initiation of therapy and exhaust limited tumour material is being questioned. In the United States, the
FDA has adopted a nimble regulatory framework to authorise/approve NGS tumour profiling and companion diagnostic devices, expanding access of analytically validated tests for patients, while ensuring that approved CDx markers are safe and effective for identification of the correct population for the use of the corresponding therapeutic product.

Adding further complexity, the structures of healthcare systems vastly differ worldwide, each operating under different socioeconomic constraints and utilising different reimbursement strategies. In Europe, these differences have led to variability among member states with regard to access to newer, expensive anticancer drugs. In the United States, there is variability according to the policies of individual private or government payers. Coverage for off-label use of a drug by private insurance companies often requires patient-by-patient petitions. If the petition is not successful, the patient or the prescribing provider is required to pay the bill thus resulting in increasing disparities in access to care. From the societal viewpoint, the challenge will be to balance access to newer therapies, which have more uncertainties regarding their benefit-to-risk ratios, and the likelihood that broader non-trial access to off-label therapies would result in significant increases in health-care expenditures.

\section{Conference consensus: biomarkers should not be developed exclusively as part of development of an individual drug}

The variable response of cancer patients to traditional and newer targeted and immune-based cancer treatments has led to intensive efforts to identify biomarkers that are predictive of drug sensitivity and/or resistance. In cases where only a minority of patients with a particular cancer subtype are predicted to benefit, CDx have been developed in parallel with newer targeted therapies and used as entry criteria for the definitive, often randomised, clinical trials that have resulted in regulatory approval. These $\mathrm{CDx}$ are then typically marketed at the same time as drug approval and designated as a gold standard for determining whether an individual patient should be treated with a specific anticancer drug. However, this is a problematic approach for the rapid and cost-effective development and use of more predictive biomarkers.

As biomarkers predictive of drug response often represent the underlying biologic mutations or processes that drive cancer initiation and progression, routine testing for these mutational processes may have clinical utility beyond treatment selection. Predictive biomarkers may also be prognostic markers (for example HER2 amplification in breast cancer) or aid in correctly classifying tumours [4,5]. By refining and confirming disease diagnosis, tumour molecular profiling may aid in 
determining the most appropriate application of standard therapeutic approaches such as surgery, radiation therapy and the use of cytotoxic chemotherapies. Biomarkers of drug response can also provide insight into heritable cancer risk (for example pathogenic germline mutations in the BRCAl gene are associated with PARP inhibitor response and increased heritable risk of ovarian and breast cancers).

As new sequencing technologies enable simultaneous and inexpensive analysis of DNA alterations in hundreds of genes or even of the entire exome or genome, the relevance of single mutation or gene focused tests for the care of individual cancer patients has been increasingly questioned. In patients with lung cancer, therapies that selectively inhibit the epidermal growth factor receptor (EGFR), ALK, ROS1, RET, BRAF, MET, HER2 and NTRK kinases have shown significant antitumour effects in subsets of patients, whose tumours harbour drug sensitive alterations in these genes. With colon cancer, targeting the EGFR pathway has only been shown to be effective in tumours lacking activating mutations in the KRAS gene [6]. Tumour mutational burden has also been shown to be predictive of response to immune checkpoint blockade or even prognostic in some but not all tumour types. While multiple CDx could be performed to determine the status of each of these predictive biomarkers, such an approach is often less efficient than the use of a single multigene NGS assay as a diagnostic test. The latter is often likely to require less tumour tissue, which could be a critical factor in patients for whom only limited tumour tissue was available from a diagnostic fine needle aspirate. It is thus apparent to most experts that for cancer patients, single gene diagnostic platforms are unlikely to be the most cost, time and/or tissue efficient means of determining which treatment approach is most likely to be effective. Conversely, NGS-based tests may have a higher failure rate than quantitative polymerase chain reaction-based tests, their accuracy for the detection of gene amplifications, deletions and fusions may be less than that for gene mutations, and they often have longer turnaround times than methods such as IHC or FISH, delays which may be problematic in cancers, where even a short delay may negatively impact outcomes. These caveats suggest that without the rigorous clinical validation provided as part of the development of companion diagnostics, multiplexed DNA sequencing assays may in some instances fail to identify the correct patient population for administration of targeted therapies or lead to patients being inappropriately treated or not receiving timely access to therapeutic agents. Indeed, a keystone principle underlying personalised medicine is that a test, which is both analytically and clinically validated, should be used to identify the correct population. The FDA has allowed for tissue agnostic claims for MSI-H/-dMMR and for NTRK-fusions with postmarketing commitments for well-validated companion diagnostics. However, FDA cannot dictate the companion diagnostic technology that sponsors propose to support a new therapeutic. Moving from single-marker tests to tests capable of generating multiple results such as NGS thus suggest that pharma companies work closely with multiple diagnostic partners. However, these issues can be mitigated by the implementation of properly validated multiplex tests using NGS or other methods in accredited laboratories participating in external quality assessment [7].

A second informative example regards deleterious somatic and germline mutations in the BRCAl gene. Pathogenic mutations in this gene have been shown to be predictive of response to PARP inhibitors in patients with high-grade serous ovarian cancer and likely other cancer types. Such mutations are also associated with increased heritable risk of ovarian, breast, prostate, pancreas and potentially other cancer types. This association with a heritable risk has made it increasingly routine to test all newly diagnosed ovarian cancer patients for germline mutations in BRCAI and other heritable risk genes so as to guide the counseling of family members concerning their potential cancer risk. It makes little sense and does not provide value to patients or the health-care system at large to retest patients known to harbour pathogenic germline mutations in $B R C A 1$ using the approved $\mathrm{CDx}$, if they later require treatment with a PARP inhibitor. Rather, there is a need to tease out germline and tumour mutations versus tumour-only mutations when addressing heritable mutations, if the mutation is found in the tumour. In addition, there is a larger need to harmonise information, among the clinical community and test developers, regarding BRCA mutations.

These examples highlight the need to reengineer the biomarker development process as it relates to CDx and LDTs. As of 2018, a CDx approval is based on demonstration that the test result correlates with the effect of the agent in a trial population or in a bridging study that reaches back to the drug trial. If a company or provider wants approval for a new alternative predictive biomarker, the concern is they will be required to perform a similar bridging study for the new biomarker. Without the flexibility proposed in these draft guidelines, the expense of generating clinical utility data would likely reduce competition, artificially elevate the cost of diagnostic testing and likely slow innovation by delaying the adoption of newer technology platforms.

Biomarker development is becoming more frequently integrated into practice changing randomised phase III studies. Because it would be unethical to repeat such phase III trials to test the utility of new biomarkers, the field is limited in its ability to show that newer, potentially more predictive or cost-efficient biomarkers are equivalent to the CDx-biomarker integrated into the initial clinical trial. Certainly, different tests for the same biomarker could be shown to have analytical 
equivalence but that would not be the case for different biomarkers. Here, some have argued that it should be enough to show that the new diagnostic test is 'equivalent' analytically and/or biologically. In regard to DNAbased testing, there is emerging consensus that accepting local technical validity should be sufficient for certain biomarkers. The MyPathway trial [NCT02091141] has demonstrated this approach for HER2 testing [8]. Such a demonstration could be coupled with prospective RWE showing that the use of newer diagnostic platforms is associated with similar clinical benefit as older diagnostic tests. This applies to DNA-based testing as well as to other IHC tests, where it has been shown that laboratory developed tests perform as well as CDx in certain settings [9], obviating the need for strict use of a CDx-assay for patient selection in a daily practice setting, for example when using PDL1-assays.

This approach could address many inefficiencies that arise from the coupling of companion diagnostics to individual drug development efforts with although uncertainty regarding clinical validity and identification of the correct patient population remains. The development of the companion diagnostic is sometimes initiated much later than the clinical study in which the efficacy of the drug was demonstrated creating challenges for industry that is trying to comply with regulatory requirements for the companion diagnostic-assay. Further, as drug approval is often, but not always, linked to contemporaneous approval based on the approval of an analytically and clinically validated companion diagnostic, drug companies may have little incentive to show that other potentially less expensive diagnostic tests could be used for the same purpose. It should also be noted that the less expensive test might also be based on an older but not necessarily less sensitive or accurate technology for the intended purpose of the assay. Lowering the cost of a predictive biomarker test could also threaten the business model of individual diagnostic companies but may be of interest to other industry segments, e.g. other diagnostic providers or payors. For instance, in MyPathway molecular testing by various local Clinical Laboratory Improvement Amendments (CLIA) approved laboratories was acceptable and showed that patients could be accurately selected for basket studies using any CLIA NGS-based assay [8]. Another problem is that there are disincentives to conduct research that could limit the population of patients treated once a drug is on the market.

Certainly, it is a missed opportunity not to include the best active comparator in the clinical development program because this hinders evaluation of added therapeutic benefit and the development of a valid costeffectiveness model $[10,11]$. Additionally, it is difficult to correct for this missed opportunity in the postmarketing phase, and consequently, precious time is lost for patients. A better alignment of evidentiary requirements and decision-making of regulators and HTA/payers could provide patients with earlier access to evidencebased innovations.

To improve the efficiency of developing newer agents or combination therapies in mutational subsets validated as drug targets, industry stakeholders would also benefit from registries that longitudinally collect mutation and patient-linked demographic and treatment response data [12-14]. Here, patient screening is performed outside of therapeutic clinical trials under the auspices of a protocol for longitudinal collection of cancer patient data and human biological material without immediate interventional intent. Informed consent is obtained with language that allows for future unspecified use of the collected data and human biological material, provided the undefined future testing plan is approved by an ethics committee (without repeat consent).

It cannot be emphasised enough that biomarkers incorporated into daily practice should be affordable, reimbursed, analytically valid and accessible to pathologists in both academic and community-hospital practices. There thus needs to be a regulatory pathway for approval of equivalent diagnostic tests of whatever nature, DNA, protein or even morphology (tumour infiltrating lymphocytes), addressing the same outcome as the biomarker used in the trial that led to regulatory approval, after the formal approval of a companion diagnostic, so as not to artificially inflate prices and hinder innovation. However, in the experience of regulators, external quality assurance schemes and diagnostic laboratories, performance can vary significantly between different diagnostic devices, and even markers on an NGS panel may exhibit variability. This is currently exemplified for assays that quantitate TMB, where it is currently unclear how the large variety of different methods and criteria used to test TMB can be useable in a clinical trial or even daily practice setting. So, while the canon of companion diagnostics may be questioned for purposes of expediency and efficiency, there is an urgent need to increase, not decrease, the quality of biomarker testing, especially for emerging biomarkers of increasing complexity because these are critical for the selection of the correct patient in the era of personalised medicine. In the current era of laboratory accreditation and given the requirement for quality control proficiency testing schemes, which in some countries includes governmental control of performance of biomarker testing, it is not unreasonable to wonder whether single gene or mutation companion diagnostics will continue to have a future role in daily practice given the robust nature and cost-effectiveness of larger gene panels. This perspective must, however, be tempered by the reality that individual lives can be dramatically affected by a suboptimal biomarker test that may lack analytical and/or clinical validity. There is an urgent need to resolve this real conflict between the accelerating pace of technology development in terms of devices and 
the slower pace of demonstration of clinical validation for the safe and effective administration of targeted therapies. Some might suggest that where possible, biomarker development should not necessarily be tied to the development of specific drugs. However, clinical validation of the biomarker to select the appropriate drug is a keystone principle that ensures safe and effective administration of drugs in precision medicine.

This is not to say that biomarkers should not be used in clinical trials to enrich enrolment for patients considered likely to respond. NCI-MATCH is a master protocol that includes signal finding, tumour agnostic studies open to adults with refractory solid tumours, myeloma and lymphoma. Individual arms are open to patients with particular mutations or mutational profiles, and these arms open and close independently once they have completed accrual. Initially, NCI-MATCH required central diagnostic testing using an NGS panel for the first 6000 patients but more recently has allowed treatment assignment to be based on results from validated reference and academic laboratories that perform genomic screening assays such as Foundation One CDx, MSK-IMPACT, etc. $[8,14,15]$. NCI-MATCH along with France Genomique 2025, SPECTA and other similar efforts may thus help facilitate the shift from single biomarker testing to the use of multiplexed assays that can detect mutations in hundreds of genes as well as more complex molecular signatures. Another example is the drug rediscovery protocol (DRUP; NCT02925234), a prospective, non-randomised clinical trial which is looking at the effectiveness of linking genetic profiles of tumours to approved targeted anticancer drugs. Patients are identified based on a Dutch whole genome sequencing initiative.

\section{The need for innovative clinical study designs}

Clinical trials of new cancer drugs are historically focused on defining the efficacy of an individual drug or combination, yet the HTAs for government health systems and insurance companies as well as approval bodies such as FDA and EMA additionally require patient-centred end-points. It might, therefore, be prudent to have a pragmatic plan approved and implemented after 'conditional approval' to reflect patient centricity in sustainable health-care systems or at least have these bodies advise on the most appropriate endpoints from their perspectives. Further, it will be essential to involve HTA and regulatory bodies, as well as patient advocates, at the clinical trial design and data collection stages rather than only after a clinical trial has completed accrual and/or analysis.

Increasingly, oncology clinical trials include imaging response as a primary or secondary end-point. Imaging has the ability to capture 3D information and therefore, quantify intratumoural heterogeneity, a cause of treatment resistance, as well as intrapatient heterogeneity, a cause of the phenomenon of oligoresistance. Now that we are moving towards quantitative imaging, protocols need to standardise procedures for image acquisition $[16,17]$. There is also emerging consensus that it is time to revisit Response Evaluation Criteria in Solid Tumours criteria and move to a more comprehensive and automated approach to quantifying in 3D tumour response $[18,19]$. There are also new quantitative imaging features emerging such as radiomics features or functional imaging features [20].

Suboptimal study designs (e.g. end-points, comparators) often result in no or delayed approval. Better trial designs, e.g. use of the right comparator, end-point, predictive markers and selection of the most appropriate patient population are needed, especially as one considers that oncologic therapy is becoming more individualised based on biomarkers (antigen expression, mutations, genotype, etc.).

Here, one can ask, what are acceptable end-points for HTAs? While decision-makers prefer clinically relevant end-points when determining drug access, e.g. overall survival (OS), life-years gained and quality of life, drug developers and regulators are increasingly relying on surrogate end-points such as progression-free survival (PFS), objective response rate (ORR), complete remission (CR) rate or molecular response. While these latter end-points can be measured earlier, they have not always been shown to be associated with traditional endpoints such as OS or supported by clinical benefit. HTAs also often focus on relative effectiveness, costeffectiveness or added value which may be difficult to extrapolate from trials that relay on surrogate endpoints. Recent examples of this include the use of minimal residual disease levels as a surrogate for durable PFS for patients receiving daratumumab for multiple myeloma, and the use of ORR, CR and partial response rates as surrogate end-points for durable long-term PFS responses for patients receiving treatment with axicabtagene ciloleucel.

The advent of personalised medicine has resulted in increasing fragmentation of patient populations, and there is thus a need to access and test large numbers of patients (even in geographically widely dispersed and varied regions around the globe) to identify sufficient numbers of patients to enrol in adequately powered studies. Furthermore, the smaller the data set, the less comprehensive the data and the closer we move towards a situation where drugs are judged based upon their effects in individual patients. Selected academics may have access to ideal patients and novel drugs, and while not denying there may be activity, the quality and the quantity of the effect needs to be assessed in broader, potentially more diverse, populations by having a larger and well-defined denominator of greater patient numbers. In most instances, it is premature for regulators to base approval decisions on the outcomes of therapeutic experiments done on few 
patients outside of controlled clinical trials, though perhaps this could become more feasible as RWE methodologies become more developed. Innovative clinical trial designs are needed that allow patients in community settings access to investigational therapies as part of a trial rather than as off-label use. For example, the AcSé program in France was designed to evaluate targeted therapies or immunotherapies in a subset of patients not enrolled in pivotal trials and to generate clinical data to support future approvals [21]. The use of a drug in a patient outside of a clinical trial or in situations when data collection is not required is often viewed as a lost opportunity to gather data that may help guide the treatment of future patients or by HTAs as a guide to how best to allocate limited healthcare resources. In sum, non-trial use of drugs should be limited by finding ways to allow oncologists who lack effective alternative treatments to try nonstandard therapies within a trial setting or, as a minimum, all non-trial use of drugs in non-standard settings should be monitored in registries that allow data collection, sharing, and analyses for a broader community and society. And, in any case, even limited data should be collected and reported.

The time taken until innovative cancer drugs are available is often unsatisfactorily long and varies considerably among the EU member states as well as between other regions in the world. In Europe, for example, oncology drugs are reviewed under a centralised procedure (EMA). The benefit-to-risk ratio is the basis for approval and marketing application authorisation applies to all member states [22]. For a member state to reimburse drugs, evaluation by an HTA is often required, whereby consideration is placed on reimbursement issues and relevance for the healthcare system, i.e. therapeutic added value and cost effectiveness. HTA agencies vary by country and often need to consider long-term and societal effects of novel drugs. Such aspects are often not included in the design of clinical trials and are often overlooked by regulatory agencies. However, despite several group efforts, EU member states, with more than 40 national and/or regional HTAs, still assess benefits and negotiate drug prices independently. Indeed, there is considerable variation in the time between marketing authorisation by EMA and HTA decisions, and there are also often differing HTA outcomes for the same drug [23]. Alignment of HTA assessments in Europe is therefore a worthy goal, and pilot projects are ongoing (e.g. EUnetHTA is a voluntary collaboration of European HTA agencies with a focus on harmonisation of HTA assessment criteria). Moreover, there are several ongoing projects to align evidentiary requirements throughout the product lifecycle between the EMA and EUnetHTA [24]. However, it has to be kept in mind that efforts to shorten the time for marketing approval via an accelerated or provisional approval process for new drugs with outstanding activity that address unmet medical needs must be balanced with the risk of harming patients should unanticipated or rare toxicities become apparent following broader clinical use.

The extent to which harmonisation is even possible or desirable also needs to be explored. In some countries, companies that make their investigational products available to patients outside of clinical trials are allowed to charge a fee, whereas in others, they are not. Another inconsistency is which entity, patient, company or health system pays for the screening necessary to detect actionable biomarkers. Patient requests for products that may help them are further complicated by off-indication requests, pediatric requests when all data was obtained in adults and requests from countries where regulatory approval, for whatever reason, was not sought. Physicians who are willing to seek non-trial access to investigational or off-label drugs for their patients face many challenges because different companies may have different policies, different countries have different procedures and in some countries, there may be legal or liability concerns. Furthermore, in all of these settings, it must be determined how data will be collected from these patients so that their outcomes and potential adverse events are not lost to history but rather used to the benefit of future patients.

The need remains to generate effectiveness data sets relevant for HTA. It is not yet clear who is responsible for these data nor whose responsibility it is to address uncertainty in these data sets. Further, how can the data be made relevant for each individual health-care system and still be generalisable so that redundant trials are not required? Programs such as SPECTA, MyPathways, and NCI-MATCH can help collect the needed data because industry cannot be expected to be able to do this alone.

Although challenges remain, all stakeholders need to come together and overcome differences between the patient-centric views of clinicians and the societycentric views of regulatory authorities and payers. There is a marked need to bridge the gap between individualised oncology, which does not currently take into account the resource constraints of health-care systems and population-based oncology, which often neglects the needs of rare cancer patients and increasingly rare molecular subtypes of common cancers. Small regulatory data sets are less useful to HTA, and efforts to use RWE to develop therapeutic guidelines need further methodologic refinement to be useful for HTA decisions. Countries, payers, academia and industry should support treatment optimisation and optimal patient selection at an international scale that will not delay patient access. All stakeholders will need to be pushed to their limits to bridge the individual-societal oncology dichotomy. 


\section{Conflict of interest statement}

R.C. received stocks from BMY (from past employment). D.B.S. served as a consultant/advisory to Loxo Oncology, Pfizer, Vivideon Therapeutics and Illumina. E.V. founded and serves on the board of not-for-profit foundations (Center for Personalized Treatment; Hartwig Medical Foundation) devoted to genomics-guided precision medicine. C.S. was a consultant for and received research funding from Astellas, Bayer, Janssen and Sanofi, received research funding from Dendreon and was a consultant for Amgen, Genentech/ Roche, Pfizer and Tolmar. R.S. reports research funding from Merck, Roche and Puma, as well as travel funds from Astra Zeneca, Roche, Merck and an advisory role for BMS, outside the scope of this work. R.S. is supported by a grant from the Breast Cancer Research Foundation (BCRF 17-194). All other authors declared no conflicts.

\section{Funding}

The Innovation and Biomarkers in Cancer Drug Development (IBCD) 2018 conference received unrestricted educational grants form AstraZeneca LP; Bristol-Myers Squibb Company; Janssen Research \& Development; Roche Holding Ltd.

\section{Acknowledgements}

The views represented in the article represent the views of the individual authors and not necessarily those of the different organisations of the faculty to whom the authors belong. Medical writing assistance was provided by John Bean PhD, Bean Medical Writing, Halle, Belgium and was funded by EORTC. The IBCD 2018 conference was managed by Davi Kaur, Danielle Zimmerman, Said Laghmari, Laurence Decroix, Frederic Rince and Gwydion Lyn of the EORTC Communication and Events Team, Brussels, Belgium.

\section{Addendum. IBCD conference faculty}

Jan Bogaerts, EORTC (Brussels, Belgium); Renzo Canetta, Independent Consultant (USA); Herbert K. Lyerly, Duke University Medical Center (Durham, North Carolina, USA); Laura Yee, NCI (Bethesda, MD, USA); David B. Solit, Memorial Sloan Kettering Cancer Center (New York, NY, USA); David Rimm, Dept. of Pathology, Yale University School of Medicine (New Haven, CT, USA); Alison Bateman-House, Division of Medical Ethics, NYU Langone Health (New York, NY, USA); Amr Makady, Interne Geneeskunde Sector Zorg Zorginstituut Nederland (Diemen, The Netherlands); Lothar Bergmann, Ambulantes Krebszentrum (Frankfurt, Germany); Sumimasa Nagai,
Translational Research Center, The University of Tokyo Hospital and PMDA (Tokyo, Japan); Marlene Thomas, Roche (Basel, Switzerland); Ian A Cree, IARC (Lyon, France); Shirley Hopper, Medicines and Healthcare products Regulatory Agency (London, United Kingdom); Chris Smith, CRUK Cambridge Institute, University of Cambridge (Cambridge, United Kingdom); Mark Robson, Memorial Sloan Kettering Cancer Center (New York, NY, USA); Mary Savage, Merck (Whitehouse Station, NJ, USA); Emile Voest, The Netherlands Cancer Institute (Amsterdam, The Netherlands); Christopher Sweeney, Dana Farber Cancer Institute, Harvard Medical School (Boston, MA, USA); Magnus Ingelman-Sundberg, Karolinska Institut (Sweden); Francois Maignen, NICE (London, United Kingdom); Benjamin Besse, Institut Gustave Roussy (Paris, France); Rafal Swierzewski (ECPC, Belgium); Philippe Lambin, Maastricht University Medical Centre (Maastricht, The Netherlands); Astrid Kiermaier, Roche Innovation Center Basel (Basel, Switzerland); Denis Lacombe, EORTC (Brussels, Belgium); Tracy Lively, National Cancer Institute (Bethesda, MD, USA); Christophe Massard, Institut Gustav Roussy (Paris, France); Roberto Salgado, (Antwerp, Belgium; Melbourne, Australia).); Vassilis Golfinopoulos, EORTC (Brussels, Belgium).

\section{References}

[1] Hayes DF, Allen J, Compton C, Gustavsen G, Leonard DGB, McCormack R, et al. Breaking a vicious cycle. Sci Transl Med 2013;5:196cm6. https://doi.org/10.1126/scitranslmed.3005950.

[2] Drilon A, Laetsch TW, Kummar S, DuBois SG, Lassen UN, Demetri GD, et al. Efficacy of larotrectinib in $T R K$ fusion-positive cancers in adults and children. $\mathrm{N}$ Engl J Med 2018;378:731-9. https://doi.org/10.1056/NEJMoa1714448.

[3] Davis C, Naci H, Gurpinar E, Poplavska E, Pinto A, Aggarwal A. Availability of evidence of benefits on overall survival and quality of life of cancer drugs approved by European Medicines Agency: retrospective cohort study of drug approvals 2009-13. BMJ 2017; 359:j4530. https://doi.org/10.1136/bmj.j4530.

[4] Duffy MJ, Harbeck N, Nap M, Molina R, Nicolini A, Senkus E, et al. Clinical use of biomarkers in breast cancer: updated guidelines from the European Group on Tumor Markers (EGTM). Eur J Cancer 2017;75:284-98. https: //doi.org/10.1016/j.ejca.2017.01.017.

[5] Nicolini A, Ferrari P, Duffy MJ. Prognostic and predictive biomarkers in breast cancer: past, present and future. Semin Cancer Biol 2018;52:56-73. https://doi.org/10.1016/j.semcancer.2017.08.010.

[6] Van Cutsem E, Cervantes A, Adam R, Sobrero A, Van Krieken JH, Aderka D, et al. ESMO consensus guidelines for the management of patients with metastatic colorectal cancer. Ann Oncol 2016;27:1386-422. https://doi.org/10.1093/annonc/mdw235.

[7] Snelson K-M, Machado JC, Rouleau E, Thunnissen E, Ligtenberg $\mathrm{MJ}$, Normanno $\mathrm{N}$, et al. Integration of nextgeneration sequencing in clinical diagnostic molecular pathology laboratories for analysis of solid tumours; an expert opinion on behalf of IQN Path ASBL. Virchows Arch 2016;470:5-20. https: //doi.org/10.1007/s00428-016-2025-7.

[8] Hainsworth JD, Meric-Bernstam F, Swanton C, Hurwitz H, Spigel DR, Sweeney C, et al. Targeted therapy for advanced solid tumors on the basis of molecular profiles: results from MyPathway, 
an open-label, phase IIa multiple basket study. J Clin Oncol 2018; 36:536-42. https://doi.org/10.1200/JCO.2017.75.3780.

[9] Kim AS, Bartley AN, Bridge JA, Kamel-Reid S, Lazar AJ, Lindeman NI, et al. Comparison of laboratory-developed tests and FDA-approved assays for $B R A F, E G F R$, and $K R A S$ testing. JAMA Oncol 2018;4:838. https://doi.org/10.1001/jamaoncol.2017. 4021.

[10] Salas-Vega S, Iliopoulos O, Mossialos E. Assessment of overall survival, quality of life, and safety benefits associated with new cancer medicines. JAMA Oncol 2017;3:382. https://doi.org/10 1001/jamaoncol.2016.4166.

[11] Gerardi C, Bertele V, Rossi S, Garattini S, Banzi R. Preapproval and postapproval evidence on drugs for multiple sclerosis. Neurology 2018;90:964-73. https://doi.org/10.1212/WNL 0000000000005561

[12] Hamid AA, Gray KP, Shaw G, MacConaill LE, Evan C, Bernard B, et al. Compound genomic alterations of TP53, PTEN, and RB1 tumor suppressors in localized and metastatic prostate cancer. Eur Urol 2018. https://doi.org/10.1016/j.eururo.2018.11. 045.

[13] Micheel CM, Sweeney SM, LeNoue-Newton ML, André F, Bedard PL, Guinney J, et al. American association for cancer research project genomics evidence neoplasia information Exchange: from inception to first data release and beyond-lessons learned and member institutions' perspectives. JCO Clin Cancer Inf 2018;2:1-14. https://doi.org/10.1200/CCI.17.00083.

[14] Cheng DT, Mitchell TN, Zehir A, Shah RH, Benayed R, Syed A, et al. Memorial sloan kettering-integrated mutation profiling of actionable cancer targets (MSK-IMPACT): a hybridization capture-based next-generation sequencing clinical assay for solid tumor molecular oncology. J Mol Diagn 2015;17:251-64. https: //doi.org/10.1016/j.jmoldx.2014.12.006.

[15] A World-Leading Molecular Insights Company | Foundation Medicine n.d. https://www.foundationmedicine.com/genomictesting/foundation-one-cdx [Accessed 19 February 2019].

[16] Lambin P, Leijenaar RTH, Deist TM, Peerlings J, de Jong EEC, van Timmeren $\mathrm{J}$, et al. Radiomics: the bridge between medical imaging and personalized medicine. Nat Rev Clin Oncol 2017;14: 749-62. https://doi.org/10.1038/nrclinonc.2017.141.

[17] Aerts HJWL, Velazquez ER, Leijenaar RTH, Parmar C, Grossmann P, Carvalho S, et al. Decoding tumour phenotype by noninvasive imaging using a quantitative radiomics approach. Nat Commun 2014;5:4006. https://doi.org/10.1038/ncomms5006.

[18] Therasse P, Arbuck SG, Eisenhauer EA, Wanders J, Kaplan RS, Rubinstein L, et al. New guidelines to evaluate the response to treatment in solid tumors. European Organization for Research and Treatment of Cancer, National Cancer Institute of the United States, National Cancer Institute of Canada. J Natl Cancer Inst 2000;92:205-16.

[19] Litière S, Collette S, de Vries EGE, Seymour L, Bogaerts J. RECIST - learning from the past to build the future. Nat Rev Clin Oncol 2017;14:187-92. https://doi.org/10.1038/nrclinonc. 2016.195.

[20] O'Connor JPB, Aboagye EO, Adams JE, Aerts HJWL, Barrington SF, Beer AJ, et al. Imaging biomarker roadmap for cancer studies. Nat Rev Clin Oncol 2017;14:169-86. https: //doi.org/10.1038/nrclinonc.2016.162.

[21] Buzyn A, Blay J-Y, Hoog-Labouret N, Jimenez M, Nowak F, Deley M-C Le, et al. Equal access to innovative therapies and precision cancer care. Nat Rev Clin Oncol 2016;13:385-93. https: //doi.org/10.1038/nrclinonc.2016.31.

[22] Bergmann L, Enzmann H, Thirstrup S, Schweim JK, Widera I, Zwierzina H. Access to innovative oncology medicines in Europe. Ann Oncol 2016;27:353-6. https://doi.org/10.1093/annonc/mdv547.

[23] Akehurst RL, Abadie E, Renaudin N, Sarkozy F. Variation in health technology assessment and reimbursement processes in Europe. Value Health 2017;20:67-76. https://doi.org/10.1016/j.jval. 2016.08.725.

[24] EMA and EUnetHTA finalise joint work plan for 2017-2020 European Medicines Agency n.d. https://www.ema.europa.eu/en/ news/ema-eunethta-finalise-joint-work-plan-2017-2020 [Accessed 19 February 2019] 